International Journal of Pure and Applied Mathematics

Volume 85 No. 1 2013, 149-154

ISSN: 1311-8080 (printed version); ISSN: 1314-3395 (on-line version)

url: http://www.ijpam.eu

doi: http://dx.doi.org/10.12732/ijpam.v85i1.12

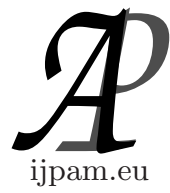

\title{
AN IMPROVED BOUND ON \\ THE POISSON-NEGATIVE BINOMIAL ERROR
}

\author{
K. Teerapabolarn \\ Department of Mathematics \\ Faculty of Science \\ Burapha University \\ Chonburi, 20131, THAILAND
}

\begin{abstract}
The Stein-Chen method is used to obtain a new non-uniform bound on the error of the negative binomial cumulative distribution function with parameters $n$ and $p$ and the Poisson cumulative distribution function with mean $n(1-p)$. The bound obtained in this study is sharper than those reported in $[8]$.
\end{abstract}

AMS Subject Classification: 62E17, 60F05

Key Words: cumulative distribution function, negative binomial distribution, Poisson distribution, non-uniform bound, Stein-Chen method

\section{Introduction}

The negative binomial distribution with parameters $n>0$ and $p \in(0,1)$ is an important discrete distribution. It is widely used in many areas of probability and statistics. For $n=1$, it is referred to as the geometric distribution with parameter $p$. Let $X$ be the negative binomial random variable with parameters

Received: February 6, 2013

(c) 2013 Academic Publications, Ltd. url: www.acadpubl.eu 
$n$ and $p=1-q$, then its probability distribution function is of the form

$$
p_{X}(x)=\frac{\Gamma(n+x)}{\Gamma(n) x !} q^{x} p^{n}, x=0,1, \ldots,
$$

and $E(X)=\frac{n q}{p}$ and $\operatorname{Var}(X)=\frac{n q}{p^{2}}$, respectively. For $\lambda=\frac{n q}{p}$ and $p=\frac{n}{n+\lambda}$, it becomes

$$
p_{X}(x)=\frac{\lambda^{x}}{x !} \frac{\Gamma(n+x)}{\Gamma(n)(n+\lambda)^{x}}\left(\frac{1}{1+\frac{\lambda}{n}}\right)^{n}, x=0,1, \ldots
$$

From (1.2), if $n \rightarrow \infty$ and $q \rightarrow 0$ while $\lambda$ remains fixed, then $p_{X}(x) \rightarrow \frac{e^{-\lambda} \lambda^{x}}{x !}$ for every $x \in \mathbb{N} \cup\{0\}$, that is, the negative binomial distribution with parameters $n$ and $p$ converges to the Poisson distribution with mean $\lambda$. In this case, some authors have tried to derive uniform bounds for the total variation distance between the negative binomial and Poisson distributions, which can be found in [3-7] and [10]. Let us consider the probability distribution function (1.1), by setting $\lambda=n q$ and $p=\frac{n-\lambda}{n}$, it can be expressed as

$$
p_{X}(x)=\frac{\lambda^{x}}{x !} \frac{\Gamma(n+x)}{\Gamma(n) n^{x}}\left(1-\frac{\lambda}{n}\right)^{n}, x=0,1, \ldots
$$

Observe that if $n \rightarrow \infty$ and $q \rightarrow 0$ while $\lambda$ remains fixed, then $p_{X}(x) \rightarrow \frac{e^{-\lambda} \lambda^{x}}{x !}$ for every $x \in \mathbb{N} \cup\{0\}$. Therefore, the negative binomial distribution with parameters $n$ and $p$ also converges to the Poisson distribution with mean $\lambda=n q$ when $n$ is large and $q$ is small. In the case of $n \in \mathbb{N}$, Teerapabolarn [8] gave a non-uniform bound on this convergence as follows:

$$
\left|\mathbb{N B}_{n, p}\left(x_{0}\right)-\mathbb{P}_{\lambda}\left(x_{0}\right)\right| \leq\left(e^{\lambda}-1\right) \min \left\{1, \frac{1}{p\left(x_{0}+1\right)}\right\} q,
$$

where $\mathbb{N B}_{n, p}\left(x_{0}\right)=\sum_{j=0}^{x_{0}} \frac{\Gamma(n+j)}{\Gamma(n) j !} q^{j} p^{n}$ and $\mathbb{P}_{\lambda}\left(x_{0}\right)=\sum_{j=0}^{x_{0}} \frac{e^{-\lambda} \lambda^{j}}{j !}$ are the negative binomial and Poisson cumulative distribution functions at $x_{0} \in \mathbb{N} \cup\{0\}$. In this study, we focus on improving the bound in (1.4) to be more sharper for any positive real number $n$.

The Stein-Chen method is important tool for giving the main result as mentioned in Section 2. In Section 3, we use this method to obtain a nonuniform bound for this error. Concluding remarks are presented in the last section. 


\section{Method}

Stein's method was first introduced by Stein [7]. The version appropriate for the Poisson case was first developed by Chen [1], which is referred to as the Stein-Chen method. Following [9], Stein's equation of the Poisson cumulative distribution function with parameter $\lambda>0$ is of the form

$$
h_{x_{0}}(x)-\mathbb{P}_{\lambda}\left(x_{0}\right)=\lambda f_{x_{0}}(x+1)-x f_{x_{0}}(x),
$$

where $x_{0}, x \in \mathbb{N} \cup\{0\}$ and function $h_{x_{0}}: \mathbb{N} \cup\{0\} \rightarrow \mathbb{R}$ is defined by

$$
h_{x_{0}}(x)=\left\{\begin{array}{lll}
1 & \text { if } & x \leq x_{0} \\
0 & \text { if } & x>x_{0}
\end{array}\right.
$$

and

$$
f_{x_{0}}(x)= \begin{cases}(x-1) ! \lambda^{-x} e^{\lambda}\left[\mathbb{P}_{\lambda}(x-1)\left[1-\mathbb{P}_{\lambda}\left(x_{0}\right)\right]\right] & \text { if } x \leq x_{0} \\ (x-1) ! \lambda^{-x} e^{\lambda}\left[\mathbb{P}_{\lambda}\left(x_{0}\right)\left[1-\mathbb{P}_{\lambda}(x-1)\right]\right] & \text { if } x>x_{0} \\ 0 & \text { if } x=0\end{cases}
$$

Note that $f_{x_{0}}(x) \geq 0$ for every $x \in \mathbb{N} \cup\{0\}$.

Lemma 2.1. For $x_{0} \in \mathbb{N}$, then the following inequality holds:

$$
\sup _{x \geq 2} f_{x_{0}}(x) \leq \frac{2 \lambda^{-2}\left(e^{\lambda}-\lambda-1\right)}{x_{0}+1} .
$$

Proof. Following [9], we have $f_{x_{0}}(x) \leq f_{x_{0}}\left(x_{0}+1\right)$ for every $x \in \mathbb{N}$. Thus,

$$
\begin{aligned}
f_{x_{0}}\left(x_{0}+1\right) & =x_{0} ! \lambda^{-\left(x_{0}+1\right)} e^{\lambda} \mathbb{P}_{\lambda}\left(x_{0}\right)\left(1-\mathbb{P}_{\lambda}\left(x_{0}\right)\right) \\
& \leq \frac{1}{x_{0}+1}+\frac{\lambda}{\left(x_{0}+1\right)\left(x_{0}+2\right)}+\frac{\lambda^{2}}{\left(x_{0}+1\right)\left(x_{0}+2\right)\left(x_{0}+3\right)}+\cdots \\
& \leq \frac{1}{x_{0}+1}\left\{1+\frac{\lambda}{3}+\frac{\lambda^{2}}{12}+\cdots\right\} \\
& =\frac{2 \lambda^{-2}\left(e^{\lambda}-\lambda-1\right)}{x_{0}+1} .
\end{aligned}
$$

Hence, the inequality (2.3) holds.

Lemma 2.2. Let $x_{0} \in \mathbb{N}$ and $\lambda=n q$, then we have the following:

$$
\mathbb{P}_{\lambda}\left(x_{0}\right)-\mathbb{N B}_{n, p}\left(x_{0}\right) \leq 1-e^{\lambda} p^{n} .
$$


Proof. $\mathbb{P}_{\lambda}\left(x_{0}\right)-\mathbb{N B}_{n, p}\left(x_{0}\right)=e^{-\lambda} \sum_{k=0}^{x_{0}} \frac{\lambda^{k}}{k !}-p^{n} \sum_{k=0}^{x_{0}} \frac{\lambda^{k}}{k !}\left(\frac{n+k-1}{n} \cdots \frac{n}{n}\right) \leq$ $e^{-\lambda} \sum_{k=0}^{x_{0}} \frac{\lambda^{k}}{k !}-p^{n} \sum_{k=0}^{x_{0}} \frac{\lambda^{k}}{k !} \leq 1-e^{\lambda} p^{n}$.

\section{Result}

The following theorem shows a new non-uniform bound on $\left|\mathbb{N B}_{n, p}\left(x_{0}\right)-\mathbb{P}_{\lambda}\left(x_{0}\right)\right|$.

Theorem 3.1. For $x_{0} \in \mathbb{N} \cup\{0\}$, if $\lambda=n q$ then we have the following:

$$
\left|\mathbb{N B}_{n, p}\left(x_{0}\right)-\mathbb{P}_{\lambda}\left(x_{0}\right)\right| \begin{cases}=e^{-\lambda}-p^{n} & \text { if } x_{0}=0 \\ \leq \min \left\{1-e^{\lambda} p^{n}, \frac{2\left(e^{\lambda}-\lambda-1\right)}{\left(x_{0}+1\right) n p}\right\} & \text { if } x_{0}>0\end{cases}
$$

Proof. It is clear for $x_{0}=0$. For $x_{0}>0$, Teerapabolarn [9] showed that

$$
\mathbb{N B}_{n, p}\left(x_{0}\right)-\mathbb{P}_{\lambda}\left(x_{0}\right)=-\sum_{x=1}^{\infty} x q p_{X}(x) f_{x_{0}}(x+1)<0 .
$$

Therefore, we obtain

$$
\begin{aligned}
0 & \leq \mathbb{P}_{\lambda}\left(x_{0}\right)-\mathbb{N B}_{n, p}\left(x_{0}\right) \\
& =\sum_{x=1}^{\infty} x q p_{X}(x) f_{x_{0}}(x+1) \\
& \leq \sup _{x \geq 2} f_{x_{0}}(x) \sum_{x=1}^{\infty} x q p_{X}(x) \\
& \leq \frac{2\left(e^{\lambda}-\lambda-1\right)}{\left(x_{0}+1\right) n p}(\text { by }(2.3)),
\end{aligned}
$$

and follows from (2.4), we obtain

$$
0 \leq \mathbb{P}_{\lambda}\left(x_{0}\right)-\mathbb{N B}_{n, p}\left(x_{0}\right) \leq 1-e^{\lambda} p^{n} .
$$

Hence, from (3.2) and (3.3), we have (3.1).

Corollary 3.1. For $x_{0} \in \mathbb{N}$ and $\lambda=n q$, then the following inequality holds:

$$
\min \left\{1-e^{\lambda} p^{n}, \frac{2\left(e^{\lambda}-\lambda-1\right)}{\left(x_{0}+1\right) n p}\right\}<\left(e^{\lambda}-1\right) \min \left\{1, \frac{1}{p\left(x_{0}+1\right)}\right\} q .
$$


Proof. It can be seen that $\frac{2\left(e^{\lambda}-\lambda-1\right)}{n}=\frac{2\left\{\frac{\lambda^{2}}{2 !}+\frac{\lambda^{3}}{3 !}+\cdots\right\} q}{\lambda}=\left\{\lambda+\frac{\lambda^{2}}{3}+\cdots\right\} q<$
$\left\{\lambda+\frac{\lambda^{2}}{2 !}+\cdots\right\} q=\left(e^{\lambda}-1\right) q$. Hence the inequality (3.4) holds.

\section{Conclusion}

The non-uniform bound on the error in Theorem 3.1, determined by the SteinChen method, is an estimate for the error of the negative binomial cumulative distribution function with parameters $n$ and $p$ and the Poisson cumulative distribution with mean $\lambda=n q=n(1-p)$. With this bound, it is indicated that the result gives a good approximation when $q$ is sufficiently small. In addition, following Corollary 3.1, this bound is sharper than those reported in [8].

\section{References}

[1] L.H.Y. Chen, Poisson approximation for dependent trials, Annals of Probability, 3 (1975), 534-545.

[2] H.U. Gerber, Error bounds for the compound Poisson approximation, Insurance Math. Econom., 3 (1984), 191-194.

[3] M. Majsnerowska, A note on Poisson approximation by $w$-functions, Appl. Math., 25 (1998), 387-392.

[4] D. Pfeifer, On the distance between mixed Poisson and Poisson distributions, Statist. Decisions, 5 (1987), 367-379.

[5] M. Romanowska, A note on the upper bound for the distance in total variation between the binomial and the Poisson distributions, Statist. Neerlandica, 31 (1977), 127-130.

[6] B. Roos, Improvements in the Poisson approximation of mixed Poisson distributions, J. Statist. Plann. Inference, 113 (2003), 467-483.

[7] C.M. Stein, A bound for the error in normal approximation to the distribution of a sum of dependent random variables, Proc.Sixth Berkeley Sympos. Math. Statist. Probab., 3 (1972), 583-602.

[8] K. Teerapabolarn, A note on Poisson approximation for independent geometric random variables, Int. Math. Forum, 4 (2009), 531-535. 
[9] K. Teerapabolarn, A non-uniform bound on the Poisson-negative binomial relative error, Gen. Math. Notes, 12 (2012) xxx-xxx.

[10] W. Vervaat, Upper bound for distance in total variation between the binomial or negative binomial and the Poisson distribution. Statist. Neerlandica, 23 (1969), 79-86. 